\title{
NUMERICAL SIMULATION OF TURBULENT FLOW AFFECTED BY VORTEX GENERATORS IN STRAIGHT CHANNEL
}

\begin{abstract}
Natalie SOUCKOVA, David SIMURDA, Vaclav URUBA`
Abstract: The presented work is the next step after several experimental examinations of the vortex generator (VG) influence on flow separation occurring on a model of the NACA 63A421 airfoil with a deflected simple flap. The other purpose of this simulation is to obtain beneficial information that can be utilized for the preparation of the experimental investigation of the same configuration using Particle Image Velocimetry method (PIV) in the future. The numerical simulation was performed for one single pair and two pairs of low-profile VGs of the same size, whose heights were smaller than the boundary layer thickness. The rectangular vane type VGs in such configuration, which generates counter-rotating vortices, was examined. The behaviour of vortices produced by VG pair or pairs in several positions downstream the VGs is investigated and will be used as a background of the measurement.
\end{abstract}

\section{INTRODUCTION}

Widespread usage and the extensive experimental research of vortex generators (VGs), which has not brought yet any generalized method of suitable VGs selection for particular cases based on principles of VGs operating, were the reasons for starting the project focused on this area of the flow control, so called passive flow control.

As it was turned out at the beginning of the project that the gained results from previous researches are applicable only for the same cases and the most efficient configurations are different for every case, although the cases are very similar. Therefore it is quite difficult to find a suitable VGs configuration for the particular case based on known results. The comprehensive research, which would bring information not only about the most effective configurations, but also about reasons, which cause that these configurations are so successful with respect to all variable parameters, could be helpful. However, performing such investigation would be severely challenging. Therefore the target of this project is to fill gaps in experimental research as well as to try to find some useful approach in the VGs selection in a wide range of application.

This research started with implementing certain VGs configuration based on outcomes of other authors on a model of a NACA 63A421 airfoil with a simple deflected flap of 20 degrees. The details about vortex generator designing and measurements are in [1] and here only the main conclusions, which define the other direction of interest, will be mentioned. The results from several visualization methods and lift slope

\footnotetext{
- Natalie Souckova, Institute of Thermomechanics, Academy of Sciences of the Czech Republic, Dolejskova 1402/5, 18200 Praha 8, Czech Republic, natalies@it.cas.cz, simurda@it.cas.cz, uruba@it.cas.cz
}

This is an Open Access article distributed under the terms of the Creative Commons Attribution License 2.0, which permits unrestricted use, distribution, and reproduction in any medium, provided the original work is properly cited. 
evaluation show differences in the VG's efficiency when the shape of the VGs is changed as well as at modification of spacing between VG pairs. Moreover, the efficiency corresponding to spacing change was opposite when the shape or size of VGs was altered, it means that large VGs with rectangular shape had greater efficiency for small spacing, VGs with triangular shape were more efficient with large spacing and the small rectangular VGs behaved the same as the triangular ones. In contrary to the results of the other authors, the efficiency of small VGs was negligible. Thus the decision to visualize and evaluate the behaviour of the vortices produced by VGs arose. Before the Particle Image Velocimetry (PIV) visualization with VGs will be carried out, the measurement of the straight channel without VGs called baseline case and the numerical simulation of the baseline case and also with VGs was performed and will be discussed in this paper.

\section{EXPERIMENT}

The experiment of baseline configuration was carried out in a blow-down facility of cross section $250 \mathrm{~mm} \times 100 \mathrm{~mm}$. The boundary layer was developed on the longer side in distance $2 \mathrm{~m}$ downstream of the channel input and the flow was evaluated using TimeResolve PIV technique. The flow condition was outer flow velocity of $4.6 \mathrm{~m} / \mathrm{s}$ with less than $0.1 \%$ of turbulence intesity.

The basic characteristics of the boundary layer were evaluated at the distance $2 \mathrm{~m}$ dowstream of the channel input, such as profile of mean velocity, standart deviation, boundary layer thicknesses and shape factor, see Table 1. More details about measurement and results are in [2]. Because the flow characteristics are known, the VGs will be placed in this position in a future measurement.

The results were used for comparison with the numerical simulation of the baseline case performed at the same conditions.

\begin{tabular}{|c|c|}
\hline$\delta_{0.995}$ & $22.5 \mathrm{~mm}$ \\
\hline$\delta_{2}$ & $2.32 \mathrm{~mm}$ \\
\hline $\mathrm{H}_{12}$ & 1.53 \\
\hline
\end{tabular}

Table 1: Flow characteristic

\section{Vortex GenERATORS}

Several different configurations and dimensions of VGs were designed for the future PIV measurement, however only three configurations were used for this first part of numerical simulations. The vane type VGs with rectangular shape were chosen and the relative position of one vortex generator to the other within a pair was such that counterrotating (CtR) vortices are produced, as shown in Figure 1. All VG's parameters are summarized in Table 2. 


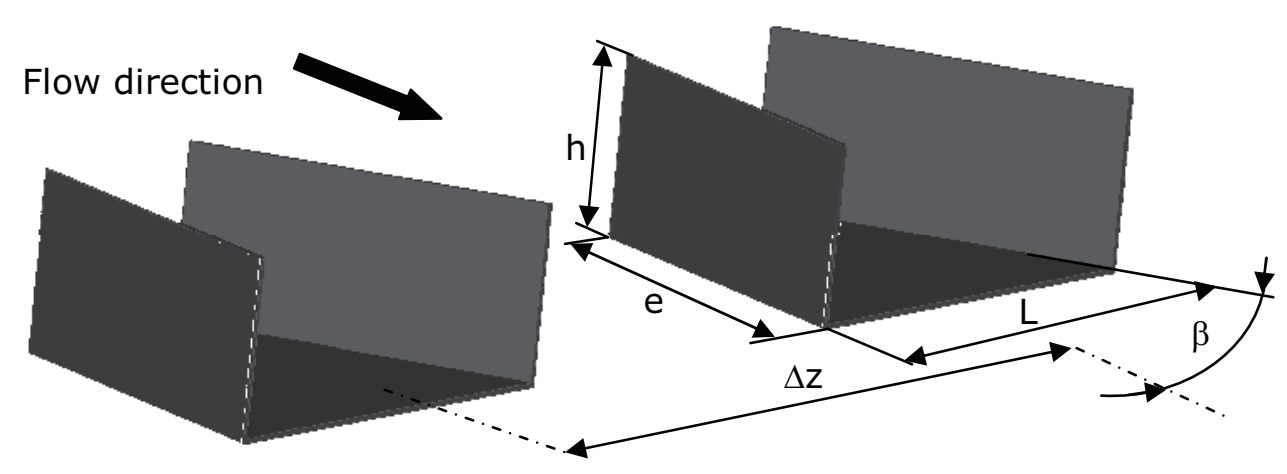

Figure 1: Configuration of VGs

\begin{tabular}{|c|c|c|c|c|c|c|c|}
\hline Type & Shape & Relative position & $\mathrm{h} / \delta$ & $\mathrm{e} / \mathrm{h}$ & $\mathrm{L} / \mathrm{h}$ & $\Delta / \mathrm{h}$ & $\beta$, deg \\
\hline vane & rectangular & CtR & 0.45 & 2 & 2.5 & - & 18 \\
\hline vane & rectangular & CtR & 0.45 & 2 & 2.5 & $3 ; 5$ & 18 \\
\hline
\end{tabular}

Table 2: Dimensionless parameters of VGs

\section{Numerical Simulation}

Based on the dimensions of the channel in the experiment, the same geometry and computational mesh for the numerical simulation of the baseline case was created with Gambit commercial software. After validating the baseline case, three other geometries and meshes with implementation of vortex generators in the middle of channel were made. The structural mesh which consists of hexahedral cells was used for all cases. The total mesh size for particular cases is in Table $\mathbf{3}$. The sample of the created geometry and mesh is shown in Figure 2.

\begin{tabular}{|c|c|}
\hline Case & Number of cells \\
\hline Baseline & 11775000 \\
\hline One pair of VGs & 12353500 \\
\hline Two pairs of VGs with spacing 3h & 11880500 \\
\hline Two pairs of VGs with spacing 5h & 11578000 \\
\hline
\end{tabular}

Table 3: Mesh size for particular cases

The numerical simulation was performed with Fluent 13.0.0 commercial code. Solutions were obtained under the assumptions of steady, three-dimensional, incompressible viscous flow. Turbulence was modeled by two-equation $k-\omega$ Shear-Stress Transport (SST) model, so fully turbulent flow was expected. The inlet boundary conditions, which correspond to those in experiment, are defined using the free stream velocity, $v_{\infty}$, the turbulence intensity, Tu, and the length scale, Ls, the values of which are presented in Table 4. Wall conditions were set on all sides of channel and on vortex generators, the pressure-outlet boundary condition was defined at the outlet of the channel, and velocity-inlet boundary condition was used for the inlet part. A second-order, upwind discretization scheme was selected with respect to the mesh used. 

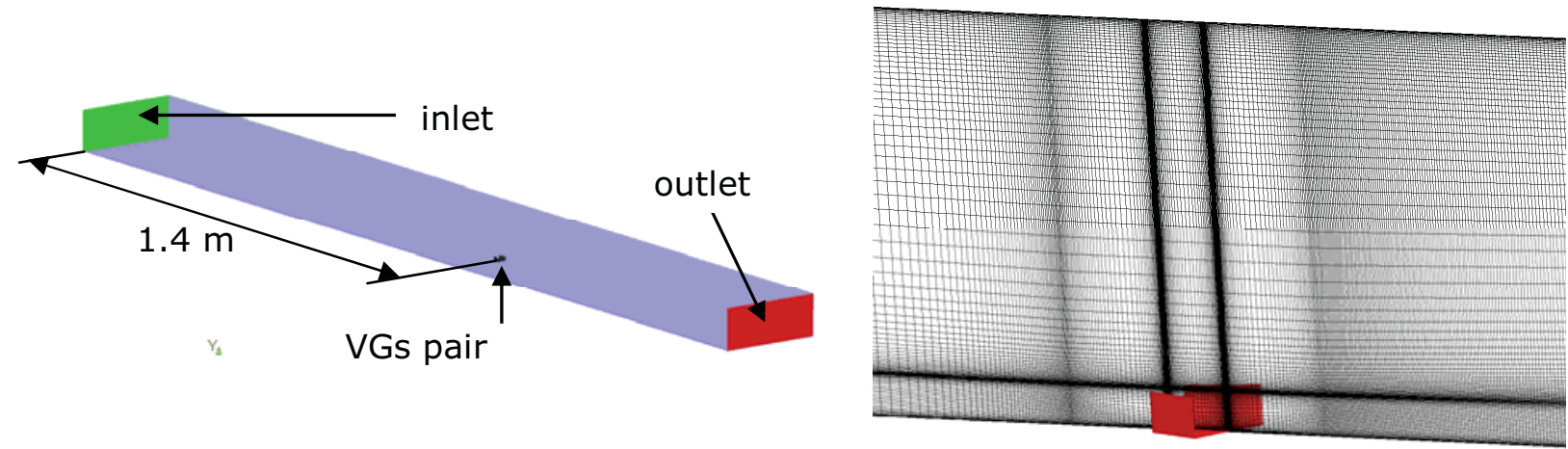

Figure 2: Samples of geometry and mesh

\begin{tabular}{|c|c|c|}
\hline $\mathrm{v}, \mathrm{m} / \mathrm{s}$ & $\mathrm{T}, \mathrm{\%} \%$ & $\mathrm{~J} \mathrm{~s}, \mathrm{~m}$ \\
\hline 4.6 & 0.1 & 0.001 \\
\hline
\end{tabular}

Table 4: Boundary condition

The mean velocity profile and afterwards the boundary layer thickness $\delta_{0.995}$ defined as (1) were evaluated in the middle of the channel on the longer side for the baseline case.

$$
\delta_{0.995}=0.995 \mathrm{U}_{\mathrm{e}}
$$

Since the boundary layer ( $B L$ ) was thicker than the $B L$ in the same evaluated position from experimental data, the other numerical simulation was performed with other turbulence models, such as $\mathrm{k}-\varepsilon$ model and Spalart Almaras. But it did not bring any improvement, therefore at preserving the same position of $B L$ evaluation the part of channel upstream of the plane was cut, thus the distance between the channel input and the evaluation plane was only $1.4 \mathrm{~m}$. At such geometry modification, the same boundary layer thickness was achieved. The same (shorter) channel model was used for cases with vortex generators.

The VGs influence on the flow was determined at 13 planes located downstream of the vortex generators position, namely at non-dimensional distance $x / h 2,6,8,10,20,30$, $40,50,60,70,80,90$ by means of the evaluation of contours of mean velocity $U, V, W$ and circulation decay for all cases. The circulation is defined as surface integral of vorticity, see equations (2) and (3).

$$
\begin{aligned}
\Gamma & =\iint_{A} \xi \mathrm{dA}, \\
\xi & =\nabla \times \overrightarrow{\mathrm{V}}
\end{aligned}
$$

\section{RESULTS AND Discussion}

\section{a. Baseline case}

As it was mentioned above, the mean velocity profile and boundary layer thickness were determined at the middle of the channel, on the longer side. Then, the profile as well as $\mathrm{BL}$ thicknesses from the experiment and numerical simulation were compared. The agreement was achieved, when the model for numerical simulation was shorter by $0.6 \mathrm{~m}$ upstream of the evaluation plane with $B L$ thickness of numerical simulation 
$\delta_{0.995}=22,3 \mathrm{~mm}$ and $\mathrm{BL}$ thickness of experiment $\delta_{0.995}=22,5 \mathrm{~mm}$. The comparison of non-dimensional velocity profiles is shown in Figure $\mathbf{3}$ and clearly, there is a very good agreement. In Figure 3 it is also drawn VG's height corresponding with a black solid line and boundary layer thickness, which is indicated by the black dashed line.

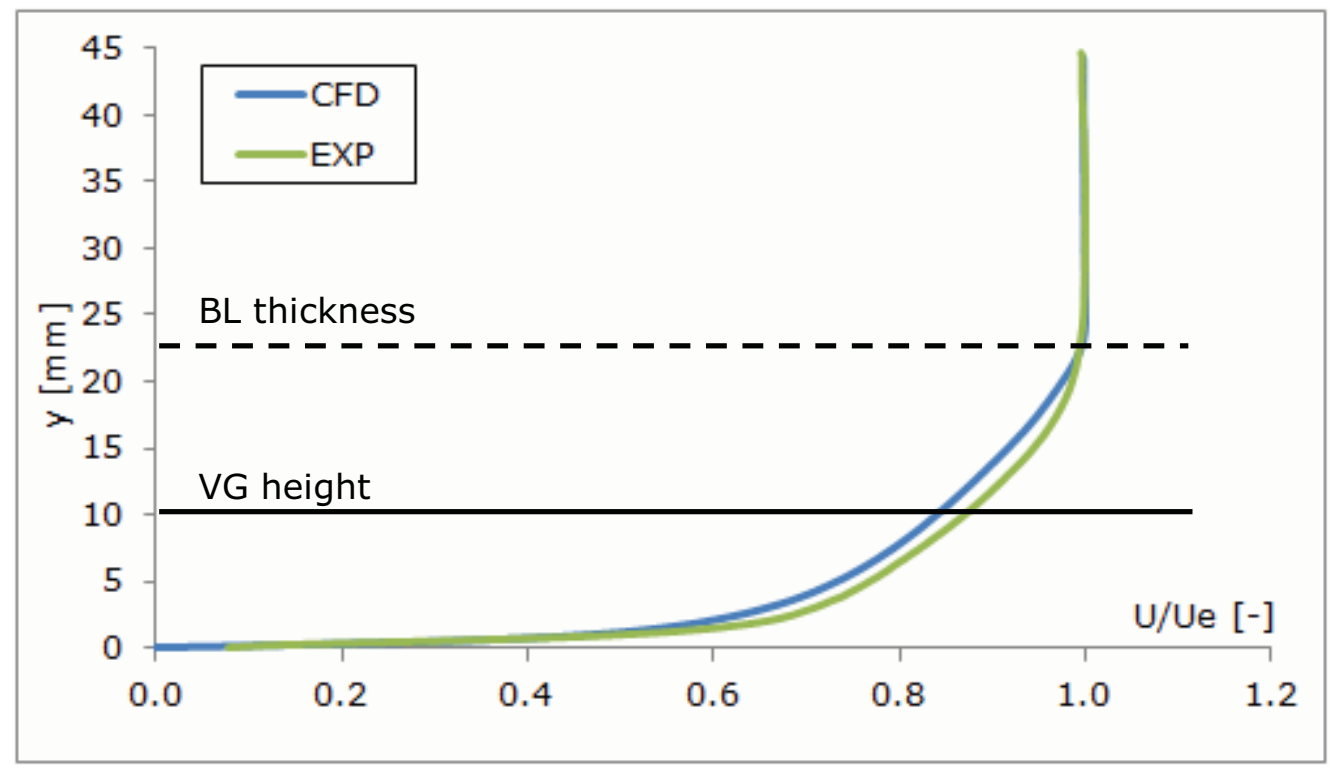

Figure 3: Profiles of mean velocity of baseline case

\section{b. One pair of vortex generators}

The results of velocity component contours $U, V, W$ from $x / h=2$ to $x / h=40$ downstream of VG pair posititon are plotted in Figure 4. Vortex generators produce two counter-rotating vortices as it was expected. All components are symetric, for $\mathrm{V}$ and $\mathrm{W}$ there is the same magnitude of negative and positive velocitites.

\section{c. Two pairs of vortex generators with spacing $3 \mathrm{~h}$}

The same evaluation like for one VG pair was made for two pairs of vortex generators and the results of velocity contours for case with $3 \mathrm{~h}$ spacing are in Figure 5. Due to vortex generators are very close to each other, there is an interaction between neighbouring vortices such as vortices merging and creating a bigger vortex, which moves up at downstream motion. Two vortices, which are not adjascent move apart at downstream motion.

\section{d. Two pairs of vortex generators with spacing $5 \mathrm{~h}$}

Figure 6 shows results of velocity contours for two pairs of VGs with a big spacing. The vortices inside are at the beginning further apart than in the case of $3 \mathrm{~h}$ spacing, nevertheless they have merged as well, but much further downstream.

The $\mathrm{V}$ component of velocity persists the longest dowstream for two VG pairs with small spacing ( $3 \mathrm{~h}$ ) and the opposite is valid for the single VG pair. The $\mathrm{W}$ component decay is the fastest for the single VG pair, but it holds out the longest dowstream for two VG pairs with large spacing (5h). These results are in an agreement with the observations in [3] as well as the results of circulation decay, see the following paragraph.

Figure 7 shows the comparison of the circulation decay for all cases. The lowest values of circulation are observed for the single VG pair. The largest circulation is achieved for 
two VG pairs with smaller spacing ( $3 \mathrm{~h}$ ) at positions close to VG trailing edge, but on the other hand this case has also the steepest circulation decay. The circulation at the end of the channel for this case is still larger than circulation of the single VG pair, but it is lower than the circulation for two VG pairs with larger spacing (5h). The circulation decay development of VG pairs with bigger spacing is similar to the one of the single VG pair. It has to be pointed out that few last evaluation planes are close to the channel outlet and the results may be affected by outlet conditions.

\section{Conclusion}

Vortex structures produced by vortex generators in turbulent flow condition were identified using numerical simulations. The components of mean velocity and circulation were evaluated in several planes downstream of vortex generators; subsequently the circulation decay assessment was made as well.

As here it is presented just the first evaluation, another one will be made in a future, consisting of vortex centre path and vortex growth evaluation. Also the other cases will be simulated such as co-rotating configuration of VG, VG with bigger size, opposite direction of VG towards the flow direction and the other changes in VG parameters. As it is mentioned at the beginning of this paper, the measurement for the same cases will be performed.

\section{ACKNOWLEDGEMENT}

The work has been supported by the Ministry of Education, Youth and Sports of the Czech Republic, within project No.1M06031. Support by the Czech Science Foundation under grants No. $101 / 08 / 1112$ is gratefully acknowledged.

\section{NOMENCLATURE}

e vortex generator length, $m$

$\mathrm{H}_{12}$ shape factor

$\mathrm{h}$ height of vortex generator, $\mathrm{m}$

$L \quad$ distance between ends of VGs in one pair, $m$

Ls length scale, $\mathrm{m}$

Tu turbulence intensity, \%

$\mathrm{U}$ component of mean velocity, $\mathrm{m} / \mathrm{s}$

$\mathrm{U}_{\mathrm{e}} \quad$ outer flow, $\mathrm{m} / \mathrm{s}$

$\mathrm{V}$ component of mean velocity, $\mathrm{m} / \mathrm{s}$

$\mathrm{v}_{\infty} \quad$ free stream velocity, $\mathrm{m} / \mathrm{s}$

$\vec{V} \quad$ velocity vector

W component of mean velocity, $\mathrm{m} / \mathrm{s}$

$\mathrm{x} / \mathrm{h}$ non-dimensional distance downstream of VGs

$\Delta \mathrm{z} \quad$ spacing between pairs of vortex generators, $\mathrm{m}$

$\beta \quad$ angle between vortex generator and axis of VGs pair, deg.

$\Gamma \quad$ circulation, $\mathrm{m}^{2} / \mathrm{s}$

$\delta_{0.995}$ boundary layer thickness, $\mathrm{mm}$

$\delta_{2} \quad$ momentum thickness, $\mathrm{mm}$

$\xi \quad$ magnitude of vorticity, $1 / \mathrm{s}$

$\nabla \quad$ nabla operator 


\section{REFERENCES}

[1] Souckova N., Kuklova J., Popelka L., Matejka M.: Visualization of flow separation and control by vortex generators on an single flap in flap configuration, Experimental Fluid Mechanics 2010, Liberec, Czech Republic, 2010, pp. 617628.

[2] Uruba V., Hladík O.: On the structure of turbulent boundary layer on smooth and rough walls, $10^{\text {th }}$ conference on Power System Engineering, Thermodynamics \& Fluid Flow - ES 2011, Pilsen, Czech Republic, 2011, pp. 1-5.

[3] Lögdberg O.: Vortex generators and turbulent boundary layer separation control, Technical reports from Royal Institute of Technology, KTH Mechanics, Stockholm, Sweden, 2006, p. 106.

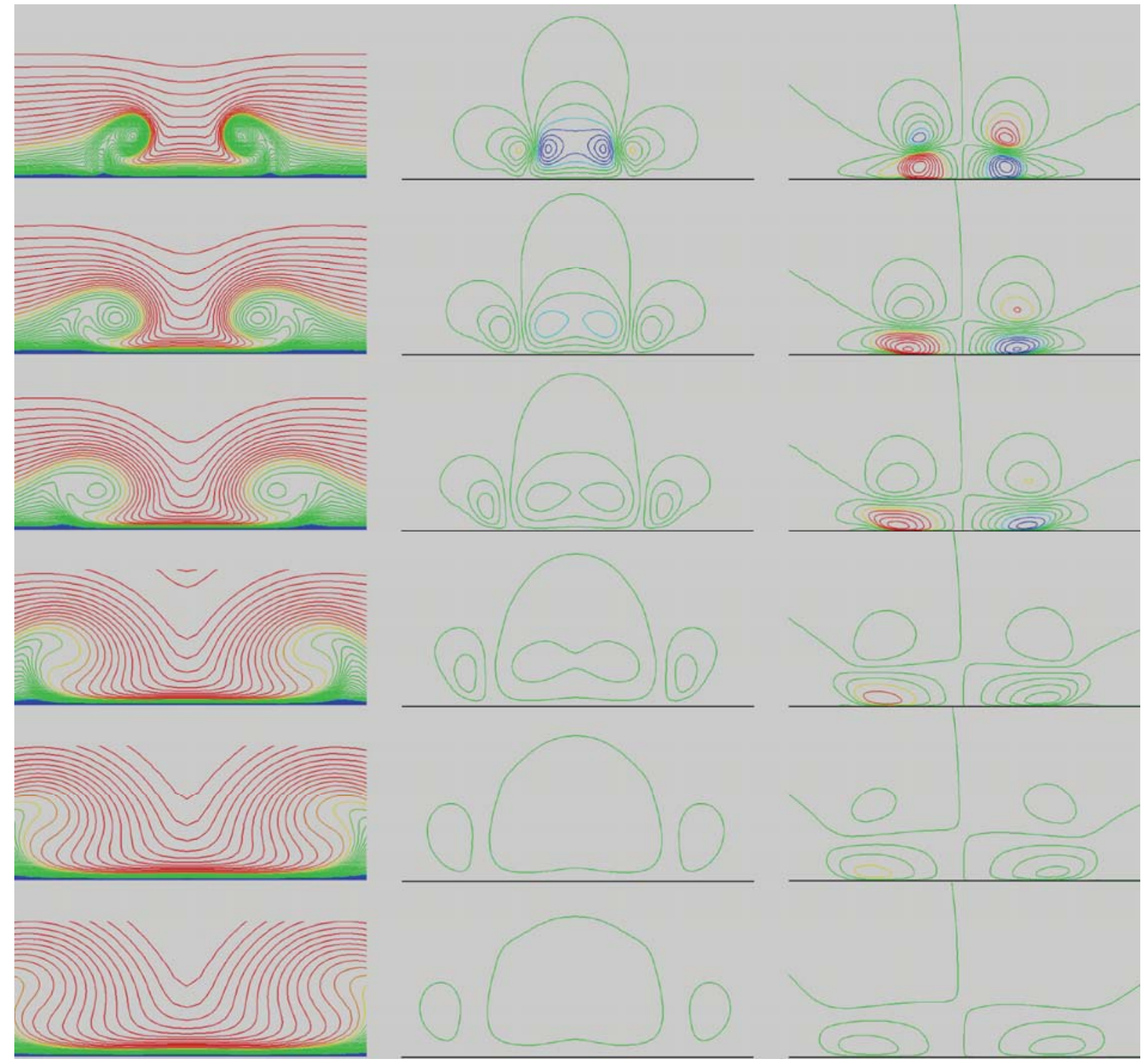

Figure 4: Contours of mean velocity for single VG pair ( $U$ - left column, V middle column, $\mathrm{W}$ - right column) at positions downstream of VG of $2 \mathrm{~h}, 6 \mathrm{~h}, 10 \mathrm{~h}$, 20h, 30h, 40h 


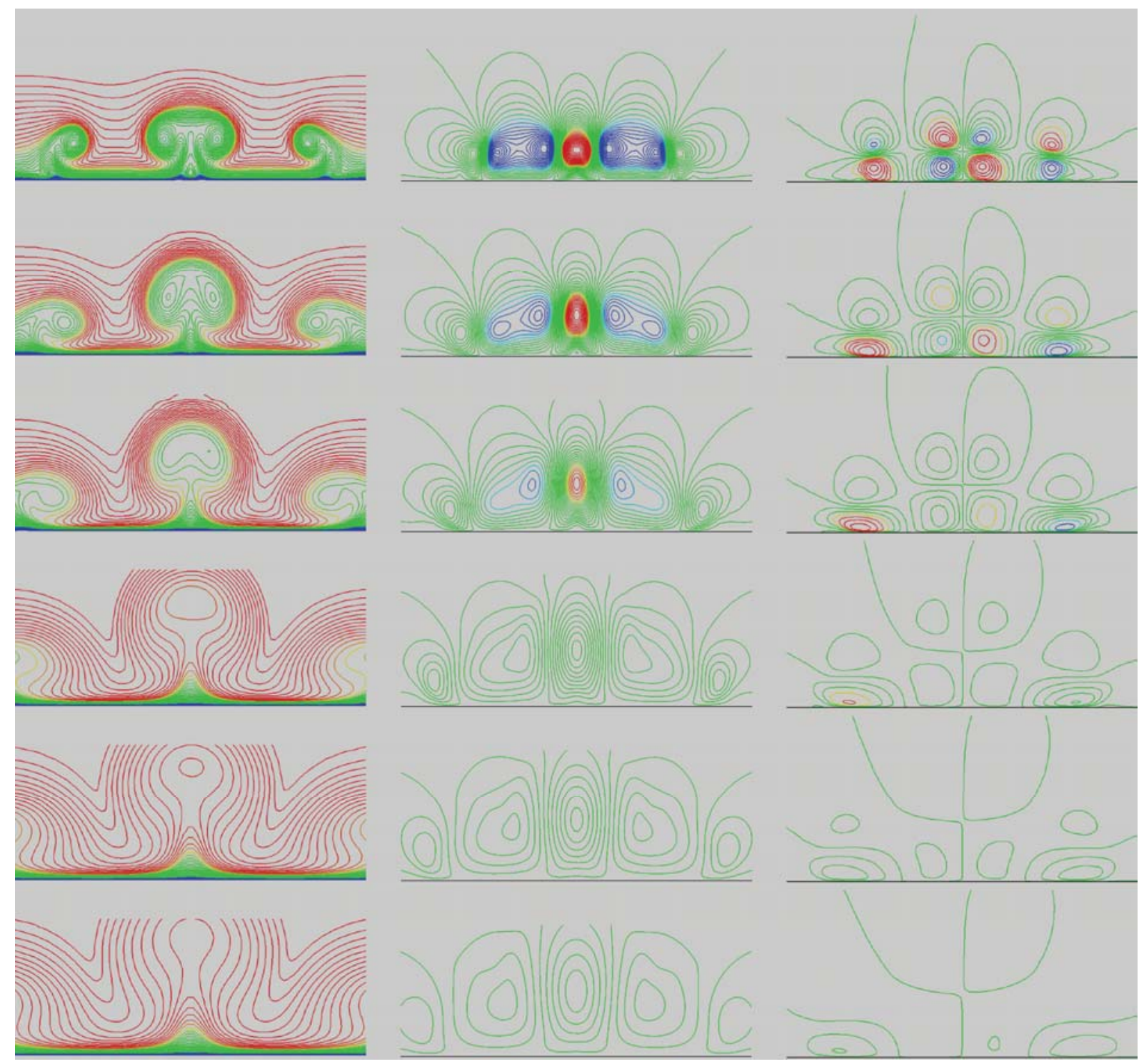

Figure 5: Contours of mean velocity for two VG pairs with spacing $3 \mathrm{~h}$ ( $U$ - left column, $\mathbf{V}$ - middle column, w - right column) at positions downstream of VG of 2h, 6h, 10h, 20h, 30h, 40h 


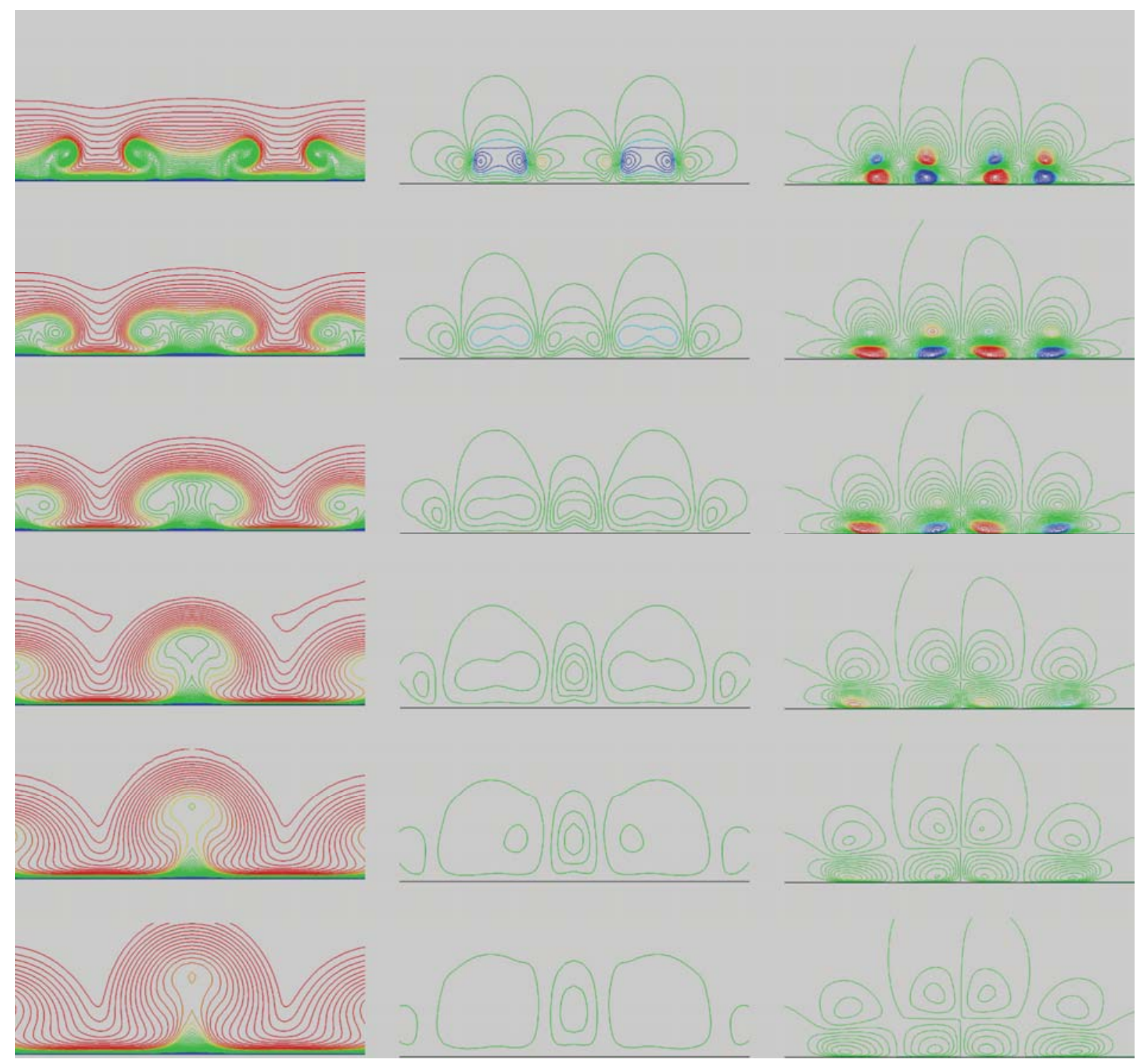

Figure 6: Contours of mean velocity for two VG pairs with spacing $5 \mathrm{~h}$ (U - left column, $\mathbf{V}$ - middle column, w - right column) at positions downstream of VG of 2h, 6h, 10h, 20h, 30h, 40h 


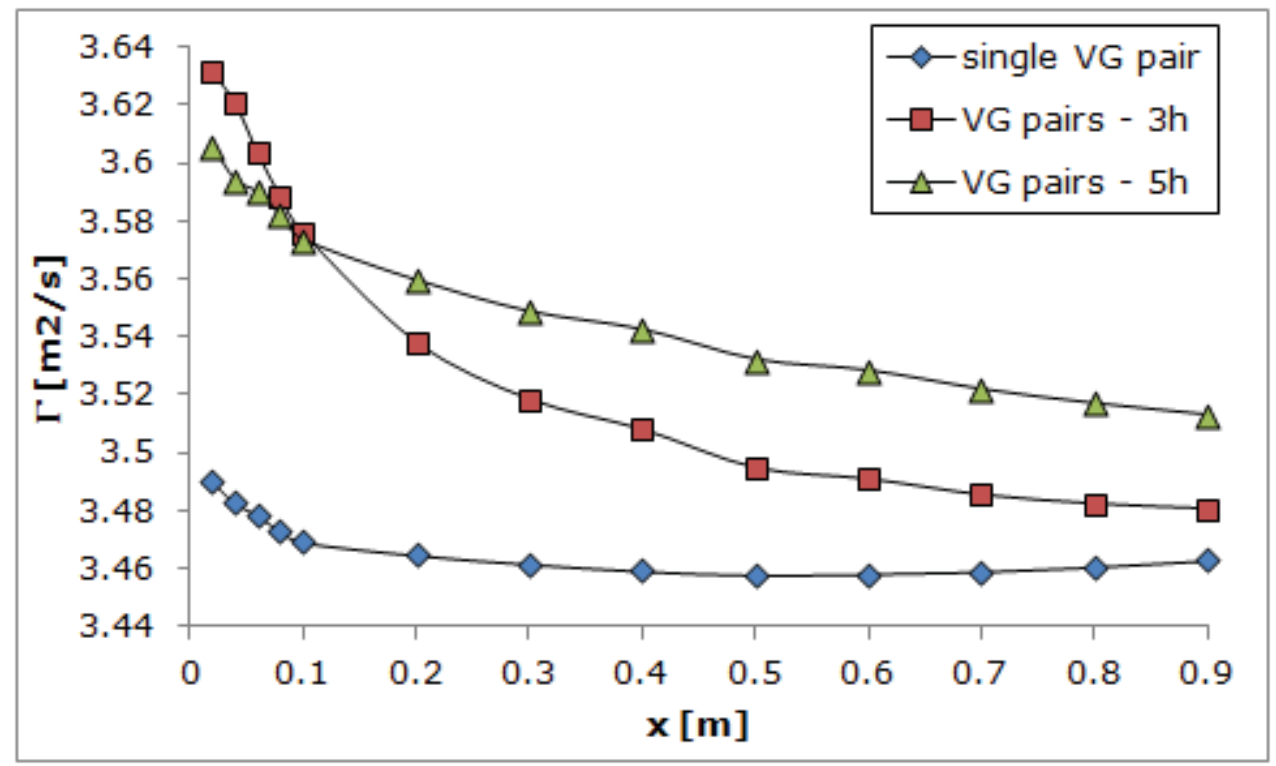

Figure 7: Circulation decay downstream of vortex generators 